\title{
Dominant Flow based Attribute Grouping for Indifferent Movement Detection in Crowd
}

\author{
Nitish Kumar \\ Indian Institute of Information Technology \\ Allahabad, India
}

\author{
Abhishek Vaish \\ Indian Institute of Information Technology \\ Allahabad, India
}

\begin{abstract}
The growth of technology is heading security systems in a challenging way with amalgamation of different threats and vulnerabilities. Although it is becoming easier day by day to find out the abnormality in the ongoing video with advancement of technology in the field of video cameras, however it is still challenging to detect the undesired event at the time of happening considering the crowd movement in a closed environment.

In the following research-paper, we propose a model which proves to be useful and applicable in detection of movement of structured crowd frame by frame in natural sequences. Evaluation results establish the fact that the proposed system is enough capable of detecting the anomalous activities such as merging, splitting or colliding at a point in a certain time than other existing techniques.
\end{abstract}

\section{Keywords}

Optical Flow, Crowd Movement, Anomaly Detection

\section{INTRODUCTION}

With the advancement of technology in each walk of life, video surveillance is becoming ubiquitous in our daily life.

Although very advanced and high-end cameras are enough capable of detecting a particular set of pattern which are predefined, yet it lacks the efficiency in certain cases which involve the tendency to deploy human-resources at each time so that this gap of detecting the anomalous pattern can be filled.

To pursue the goal of detection of certain activity which is differing from the natural, there have been an enormous research which has been proved worthy yet in real-world scenario; it becomes very compact situation either in case of individual human movement or crowd movement. One of the most promising methodologies to deal it with this challenging issue is flow based analysis of crowd movement. Tracking each and everyone in crowd creates complexity when we try to findout the optical flow of the movement of individual objects. 1

Some researchers have used computational techniques to find out the instantaneous flows of the crowd movement at a particular interval of time. 2

Flow based analysis and methodology is very promising but its relevancy is not up to mark under certain condition as crowd may be structured or unstructured and crowd movement is always a non-rigid motion. 3

Certain other approaches have been used to find out the action (predictable movement) of the crowd provided that the movement of the crowd is in structured way and it is unidirectional. 4
Detecting of next action / or finding out the abnormality in the movement of crowd is quiet challenging provided that normal pattern of the movement is pre-defined, in structured crowd movement, noise, occlusion and corner-based feature issues, creates impact when we try to compute the motion-flow.5

Some other issues we have to consider while analyzing the flow based approach of crowd movement that during the group based crowd movement, some elements of the moving crowd may stop at certain point and this represent the flow point to zero value and at the same time, there may be condition that certain activity is happening momentarily in that space, so capability of algorithm should be enough standardized to find out the differences between both condition. Some scientific approaches have tried to solve the issue of abnormality detection of crowd movement with the above discussed points. 6

To solve the problem of classical approaches of optical flow being used for anomaly defection in crowd movement which ignores the grouping of people as elements on the basis of their individual movement, we propose a computational model based on optical flow grouping of people moving in a similar direction at similar velocity.

In this paper, we have proposed a model of anomaly detection system based on low-level features of crowd movement in a closed boundary, the system is able to detect the motion of crowd moving in a particular direction and find out abnormality in it in different scenario.

This paper is organized into following sections -

Section-2 deals with "Related Work". In next Section-3, we discuss our crowd flow analysis system.

In its sub-part, 3.1 - we discuss on Optical flow Computation, Data Generation of movement based on certain attributestrajectory based data generation. Velocity based and Acceleration based flow data generation. Section 4 deals with flow density based clustering. Section 5 deals with Crowd Tracking based direction based flow grouping. (For this we calculate patch based orientation histogram). Evaluation result and conclusion has been discussed in this part. 


\section{DATA FLOW DIAGRAM}

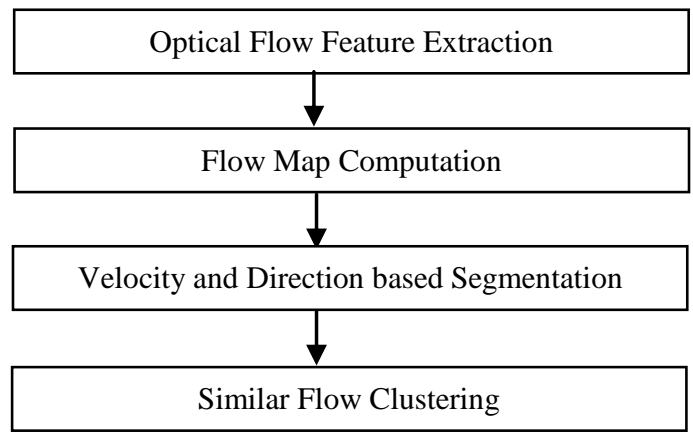

\section{RELATED WORK}

Usual event detection in crowd movement have attracted the attention of researchers from long-time and several approaches have been used involving optical flow methodology.

Tracking of crowd as a single unit based on its optical flow which is generated from its attributes has been a unique approach, such as 7, $12 \mathrm{X}$. Wang presented on approach in 2006 based on trajectories generated from velocity of crowd, in which global motion flow was analyzed to find out the movement.

Some approaches have been applied to find out the challenges in this field based on the trajectory analysis of optical-flow generated by interaction of individual person in a space-time phenomenon however in this approach different individual elements of the crowd were analyzed based on their interaction, whole crowd was not considered as an individual element. 8

Several methodologies have been used holding this idea of treating human as particle; Solmaz used this approach to find out the next happening event while Ihaddadene and Djereba used the same phenomenon considering motion intensity as a heat map data collected through attributes of velocity, direction and density. 9

Some researchers have used a new approach matching with existing techniques as they predefined the region of interest in the trajectory flow map of movement and matched the next pattern with existing match and proposed a model to predict the next action.

Some other researchers have used optical-flow to detect anomalous movement of crowd in a closed (controlled) environment by using their low-level features.10 In which they proposed a model to predict the next special bounded set of event instead of abnormal movement, going ahead SVM, HMM and Self-Organizing Map or other similar methodologies have been also used to learn the dominancy in the flow of emergence or collective gathering or dispersion of crowd.11

In 2009, Y. Benabbas and his colleagues proposed a conceptual model, for detection of major flow generated by crowdmovement in which a directional model was used to defect the event, which is constructed from Von Mises distribution over the orientation of optical flow. These approaches suffer with the challenges of motion types. Our work further extends the use of optical flow based trajectory data analysis along with algorithm which perfectly works in controlled environment of structured crowd movement. Experiments demonstrate the accuracy and effectiveness of the proposed algorithm in detecting the abnormal event in crowd movement.

\section{FRAMEWORK}

Anomaly detection in video-surveillance is a complicated procedure when it depends on different scene. In real time scenario, there can be two types of crowd in a scene-

(i) Structured Crowd

(ii) Unstructured Crowd

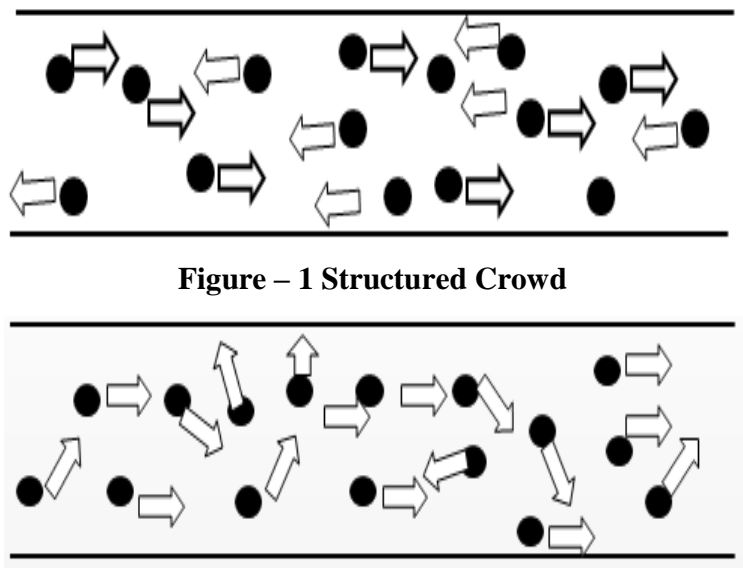

Figure - 2 Unstructured Crowd

Here "Unstructured crowd is beyond of our research motto. Structured crowd may be defined or characterized in a way where boundaries are well defined and direction of moving element is known or may be perceived easily and Unstructured crowd can be termed any type of crowd movement in which boundaries of movement direction of movement, velocity of the people moving in the crowd is uncertain and is not coherent and having different values.

When we try to address the problem of abnormality detection in a structured crowd two important attribute has to be decided-
(i) Velocity
(ii) Direction

We acquire the optical flow of the crowd in which people are moving in a structured way.

The crowd movement may have different type of anomalies depending on their context such as movement of an athlete in a wrong direction in comparison to his competitors in other lanes, or velocity of a moving person may be treated indifferent if his velocity differs with his compeers.

The main factor here is the "Context" and it has two deciding variables- Velocity and Direction.

In our research work, we have considered these two variables (velocity \& direction) for abnormal movement detection in structured crowd. Crowd has two scene based category-

\section{(A) Monovariate Movement}

\section{(B) Multivariate Movement}

Here Monovariate crowd movement stands for that type of crowd movement in which one variable is varying and another variable out of velocity and direction is fixed, And Multivariate crowd movement stands for such type of crowd movement in which either velocity is fixed but direction is not fixed.

Monovariate crowd movement has two classes on the basis of their movement-

(A) Monovariate Uni-directional movement

(B) Monovariate Bi-directional movement 


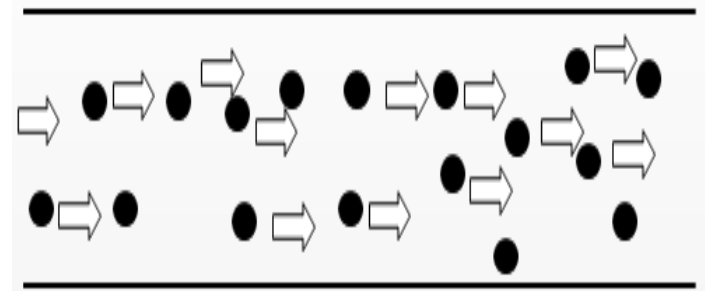

Figure - 3 Monovariate Unidirectional

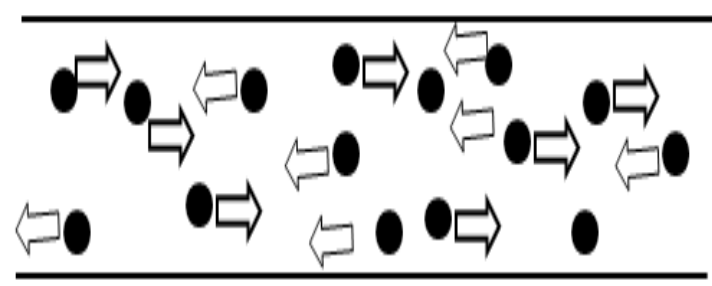

Figure - 4 Monovariate Bidirectional

So, here abnormal movement can be explained as in case of "Monovariate-Unidirectional structured crowd movement as in figure-

(i) Straight forward movement:- Which is unidirectional (in one direction)

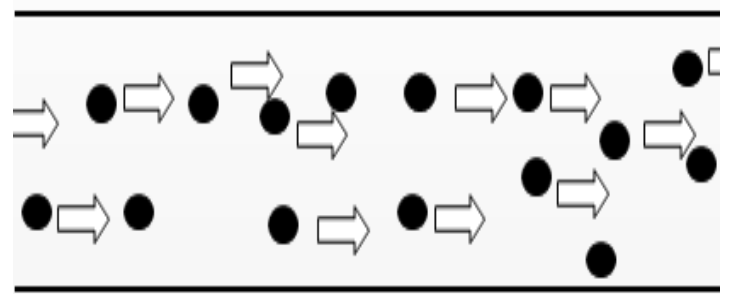

Figure - 5 Unidirectional Crowd Movements

Here in the above movement, an element can be treated indifferent to others if his movement is not like others based on three criteria-

(i) Trajectory based

(ii) Velocity based

(iii) Direction based

If trajectory or border is fixed so only two factors are responsible for deciding the indifferent movement in structured crowd movement scenario -

\section{(i) Velocity}

(ii) Direction

\section{EXPERIMENTAL WORK}

\section{Crowd - Flow Analysis System}

In a structured crowd movement, either velocity or direction or both may be fixed at a time or both may vary simultaneously but in a real-time scenario direction rarely matters and spatial clogging occurs which creates the blockage or collision like situation $^{12}$ which is treated as a chaos or stampede situation.

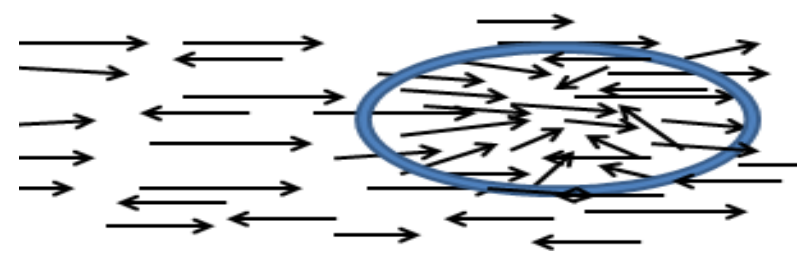

Figure - 6 Real time Flow based crowd situation
Here orientation and moving velocity is most significant information to find out the collision like situation, so we will classify the flows into two classes, one on the basis of velocity and another is direction, and to achieve this, first well acquire the image and do segmentation, after that we find out the "Orientation histogram" to exactly depict the collision region spatially.

After segmentation, flow vectors in both groups will be clustered.

\section{Optical flow Computation}

Here we have used "Lucas-Kanade algorithm to calculate the optical flow with OpenCV and after that we group these motion vector into cluster using hierarchical clustering. In motion vector which are very close to each-other, we group those all motion vector together -

\section{Optical flow}
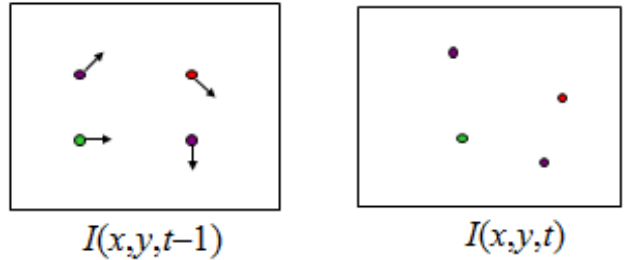

\section{Figure - 7 Optical Flow Point Demonstration}

For a given two subsequent frames, we estimate the apparent motion field between them here key assumptions are ${ }^{13}$ -

(i) Brightness is constant in both the image as spatial movement has no effect of it.

(ii) Minimal movement: elements do not move for a long0distance, and their small movement is detectable by camera.

(iii) Spatial coherence: Point has similarity in their movement based on their velocity or acceleration.

Brightness Constancy:

$$
\mathrm{I}(\mathrm{x}, \mathrm{y}, \mathrm{t}-1)=\mathrm{I}(\mathrm{x}+\mathrm{u}(\mathrm{x}, \mathrm{y}), \mathrm{y}+\mathrm{v}(\mathrm{x}, \mathrm{y}) \mathrm{t})
$$

So it can be written as:

$$
I n=\frac{\partial I}{\partial n}
$$

$\mathrm{I}(\mathrm{x}, \mathrm{y}, \mathrm{t}-1) \sim \mathrm{I}(\mathrm{x}, \mathrm{y}, \mathrm{t})+\mathrm{In} \cdot \mathrm{u}(\mathrm{x}, \mathrm{y})+$ Iy.r $(\mathrm{x}, \mathrm{y})$

So, Ix.u + Iy.v + It $\sim 0$

Selected Dataset: have we have selected three data-set.

(A) CAVIAR data-set ${ }^{14}$

(B) AGORA data-set ${ }^{15}$

(C) Our own data-set

(A) CAVIAR data-set: In this we have selected two scenarios (I) Fight Case -

(i) Two people fight and run-away

(ii) Two people meet fight and chase each other

(iii) Two people meet, fight and both run-away

(iv) Two people meet, fight and one is made down, other runaway.

(II) Walking Case:

(i) Two people meet fight \& run-away. 
(ii) Two people, meet, fight and chase-each other

(iii) Two people meet, fight and run-away both.

(iv) Two people meet, fight, one down, other run-away.

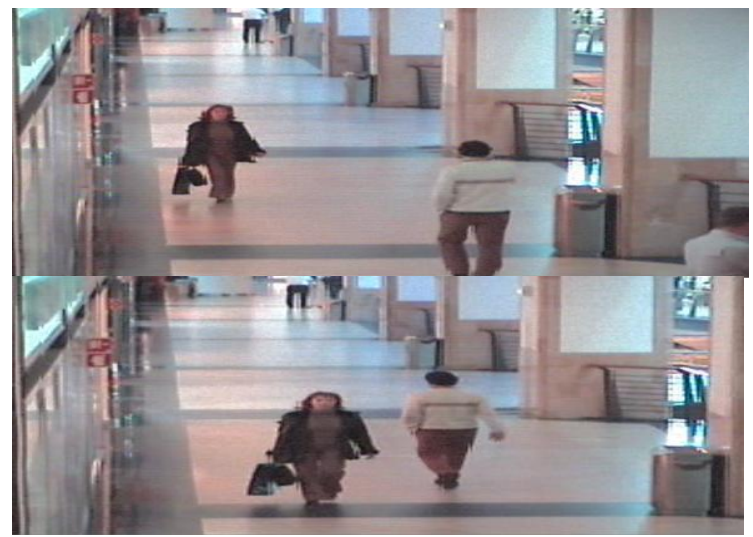

Figure - 8 CAVIAR dataset instances

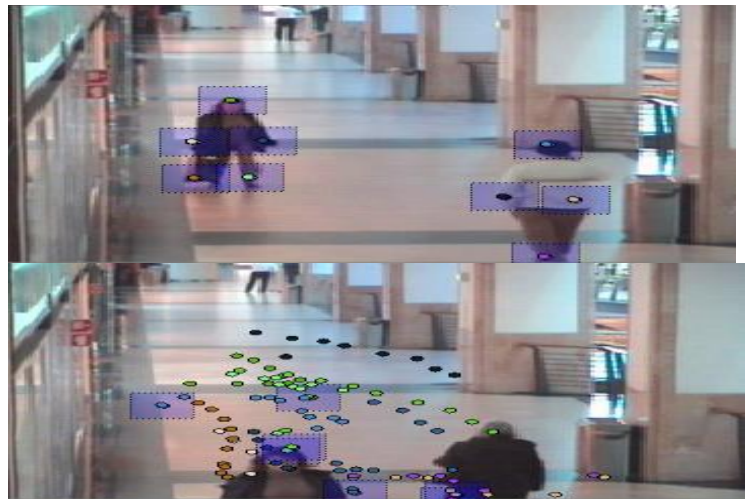

Figure - 9 Direction and Velocity based Segmentation

(B) AGORA dataset: In this we selected two cases:

(i) People walking through obstacle.

(ii) People walking in Mecca circularly.

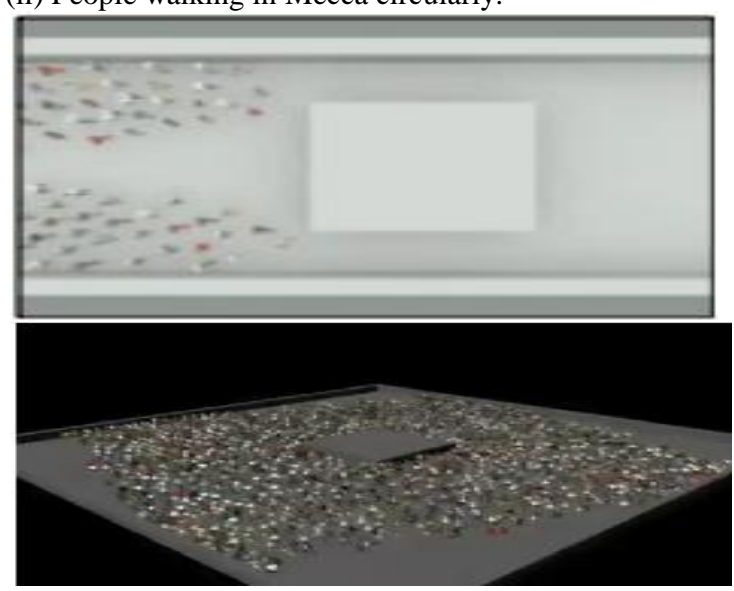

Figure - 10 Moving Structured Crowd AGORA Dataset
(C) Our Own dataset: We generated the exact dataset using our own set-up and grouped then based on their velocity and direction.
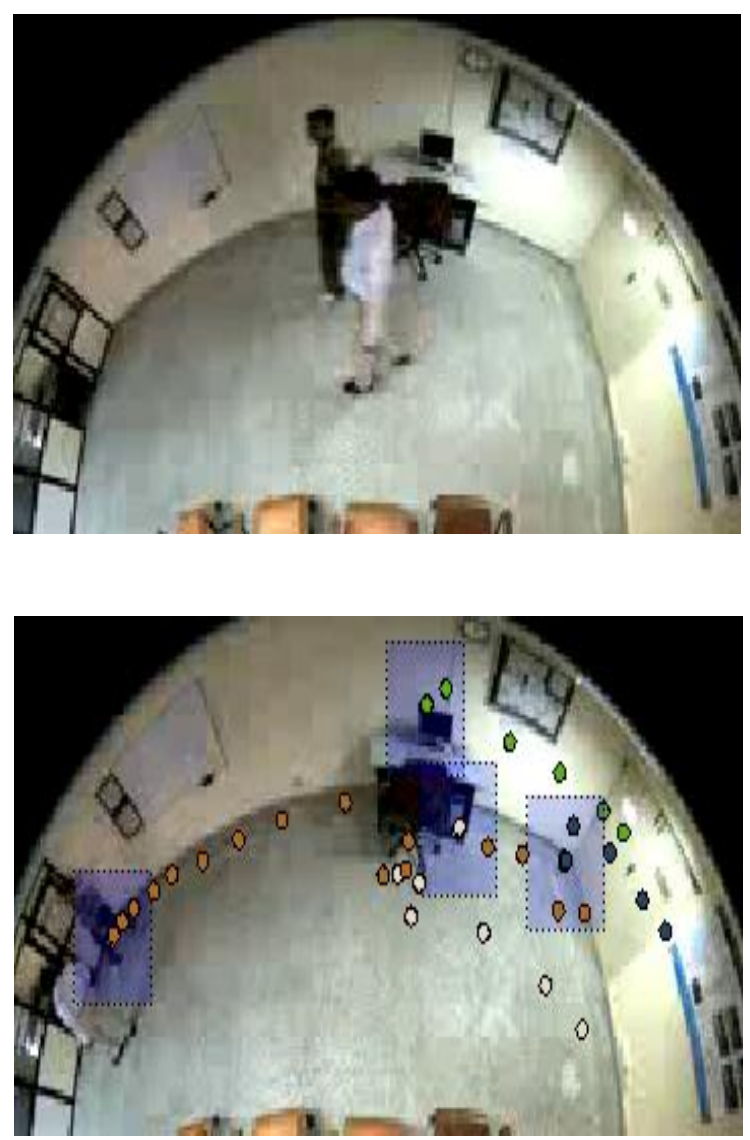

Figure - 11. Experimentation in our Dataset:
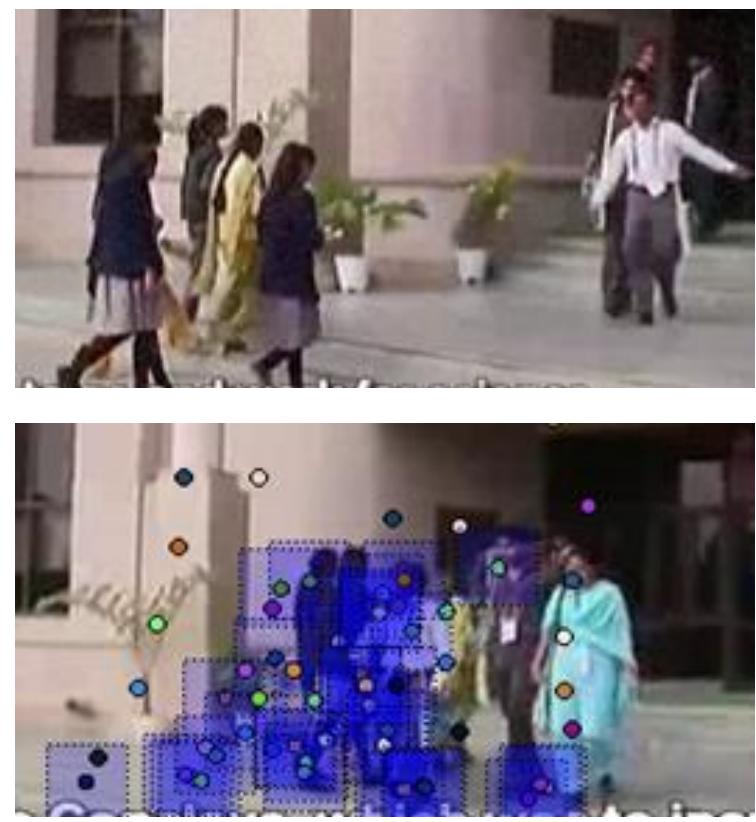

Figure - 12 Instances of crowd movement and output of experiments 
On the grouping, we find out that there are two classes in velocity based grouping-

(i) Paired Motion flow: the velocity variation in each element is in a controlled way and under the hold limit, it matches with each-other.

(ii) Un-paired Motion flow: the velocity of each element is in stochastic manner and they cannot be paired even if being directionally in equal phase.

\section{Clustering of Paired Motion flow groups:}

Once controlled pair motion flows has been groups, then we have to find the domain art flow out of the so that we can speculate the most probable positive direction of flow. Have firs the strongest flow is computed and rest of group of the flows are connected to that ${ }^{16}$.

The algorithm as follows:-

(i) Determine the connected cells with similar values.

(ii) Search the most flows which are in same direction and group it.

(iii) Find the closet set of flow group and connect it with previous one.

(iv) Find the closet neighbor between both group and group then as one group.

(v) Collect the other remaining flows which have no similar value in their cell $\mathrm{N}$.

(vi) Compare both flow and find out different size.

\section{In OpenCV}

$$
\begin{aligned}
& \text { for (int } \mathrm{h}=\theta ; \mathrm{h}<\text { cluster container }() ; \mathrm{h}++) \\
& \text { crpoint pt1, pt2 } \\
& \text { CvMat box[100] } \\
& \text { pt1.x }=\text { (int) points } \rightarrow \text { data.f }[\mathrm{h} * 2] \text {; } \\
& \text { pt2.x = (int) points }->\text { data.f1 }[\mathrm{h} * 2+1] \text {; } \\
& \text { CV circle (frame1, pt1, 20, CV_RGB, 4); } \\
& \text { And for global orientation detection - } \\
& \text { double calcglobalorientation (Input Array }
\end{aligned}
$$
orientation, InputArray Mask, InputArray MHI, doubleduration);

$$
\text { Void Opticalflow:calc(InputArray10, }
$$
InputArray I1, InputOutputArray flow) ${ }^{17}$

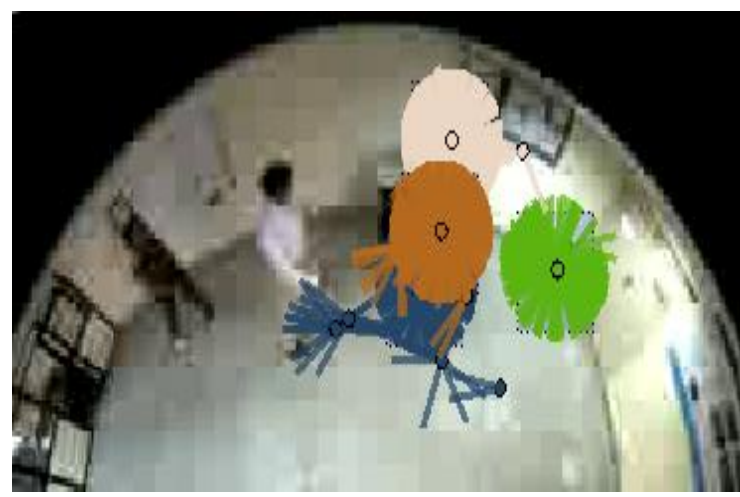

Figure 13 Average positions of circles

In desired output, most of time overlapping situation occurs, if one point describing each circle in the image, then the distance between two or more than two circles should be less than the threshold value. If two circles are close to each-other too much then we have to define a new circle at their average position of both circles last.

\section{Experimental Result and Discussion}

Our experimental work, crowd data-set taken from

(A) CAVIAR shows that (i) Variability of structured \& unstructured crowd movement difference.

(ii) It also shows the inefficiency of attributes present in CAVIAR data-set for such type of movement detection of the same, we were able up to a level, to group to similar directional vectors in this data-set.

(B) In our work, on AGORA data-set we were almost successful to find out the flow generating from one group were able to group then and we were able to apply our algorithm to re-organize the similar groups to the major group having dominant flow and were able to detect the indifferent flow, which cannot be grouped together.

(C) In our third selected dataset, which was generated using our own setup, we successfully demonstrated and showed the optional flow and direction connectivity between cells of a region. In indifferent moving scenario, we were able to demonstrate the similar element based on their controlled velocity or their matching equal phase directional movement. We also demonstrated the dominant flow with connected similar group and after that the movement based grouped unmatched with the remaining grouped element separates the normal moving crowd from abnormal moving crowd.

\section{CONCLUSION}

In this research work we presented a novel approach to find out the controlled dominant motion flow, based on two variables, velocity and direction. By using hierarchical clustering approach it is nearly possible to group the normal movement and abnormal movement. In a structured motion crowd flow. This proposed method can be used to detect some other context based abnormality relating to velocity, direction and trajectory in structured crowd movement.

\section{REFERENCES}

[1] F.M. Porikli, Trajectory Pattern Detection by HMM Parameter Space feature and Eigenvector Clustering ECCV, 2004.

[2] M.Hu, S. Ali and M. Shah, Detecting Global Motion Pattern in Complex Videos, ICPR, 2008.

[3] M. Rodnguez, S. Ali and T. Kanade, Tracking in Unstructured Crowded Scene ICCV-2009.

[4] M. Hu, S. Ali, and M. Shah, Detecting Global Motion Pattern in Video, ICPR, 2008.

[5] T.B. Moeslund, A. Hilton and V. Kruger, A survey of advances in vision-based human motion capture and analysis.

[6] E.K. Tzamali, M.D. Plumbley and S.A. Velastin Motion estimation for crowd analysis using a hierarchical Bayesian approach, London, U.K.

[7] X. Wong et al., Learning Semantic Scene Models by Trajectory Analysis ECCV-2006.

[8] R. Mehran, A. Oyama, and M. Shah, "Abnormal crowd behavior detection using Social force Model" in 2009 IEEE (Conference on Computer Vision \& Pattern Recognition-2007, pp-1-6. 
[9] N. Ihaddadene, and C. Djeraba, "Real time Crowd Motion Analysis" in ICPR 2008, 2008, pp. 1-4.

[10] L. Kratz and K. Nishino, "Anomaly detection in extremely crowded scene using spatio-temporal motion pattern models", in a CVPR, 2009, pp. 1446-1453.

[11] E. Andrade and R. Fisher, "Modeling crowd scene for event detection", Proc. of 18th International Conference on Pattern Recognition ICPR-06 Hong-Kong August 2024, 2006, pp. 175-178.

[12] M. Black and D. Fleet. Probabilistic detection and tracking of motion boundaries. Int'l. J. Comp. Vision, 38(3):231-245, 2000.

[13] James Hays (Brown); Silvio Savarese, Motion Estimation, (U. of Michigan); Octavia Camps (Northeastern), Web address

http://cronos.rutgers.edu/ meer/UGRAD/cv15motion.pdf accessed at 12/01/2014, $1400 \mathrm{hrs}$.
[14] CAIVIAR dataset, EC Funded CAVIAR project/IST 2001 37540, found at URL: http://homepages.inf.ed.ac.uk/rbf/CAVIAR/

[15] P. Allain, N. Courty, and T. Corpetti. Crowd flow characterization with optimal control theory. In ACCV, Xi'an, China, 2009.

[16] Ozturk, O., Yamasaki, T., Aizawa, K.: Detecting dominant motion flows in unstructured/structured crowd scenes. In: Proceedings of the 2010 20th International Conference on Pattern Recognition, ICPR 2010, pp. 3533-3536. IEEE Computer Society, Washington, DC (2010).

[17] Motion Analysis and Object Tracking Using Lucas Kanade Method, accessed at http://docs.opencv.org/master/modules/video/doc/motion_ analysis_and_object_tracking.html\#motion-analysis-andobject-tracking on 14 Jan, 2014 at 1600 Hrs. 